\title{
Строчтепьные конструкиU
}

\section{РАСЧЕТ ОСЕСИММЕТРИЧНЫХ ТОЛСТЫХ ПЛИТ, СВОБОДНО ОПЕРТЫХ ПО КОНИЧЕСКОЙ ПОВЕРХНОСТИ (С ВОЗМОЖНОЙ ПОДВИЖКОЙ)}

\author{
CALCULATION OF AXISYMMETRIC THICK PLATES FREELY SUPPORTED \\ ON CONICAL SURFACE (WITH POSSIBLE VERTICAL DISPLACEMENT)
}

\begin{abstract}
Приводится расчет напряженного состояния и предельной нагрузки осесимметричных толстых плит, свободно опертых по конической поверхности с возможным перемещением по вертикали. Расчетная зависимость по определению предельного давления получена с использованием уравнения равновесия для сферического купола. По предложенной методике выполнены примеры расчета толстых плит.

Ключевые слова: корпус высокого давления, торцевой элемент, толстая плита, несущая стенка, радиальные напряжения, тангенциальные напряжения, вертикальные напряжения, внутреннее давление, равномерно распределенная нагрузка, шпонка, контактный слой.
\end{abstract}

The present paper submits the results of calculation of the stress state and limiting loading of axisymmetric thick plates freely supported on conic surface with possible vertical displacement. Using the equation of equilibrium for the spherical dome, there was obtained the estimated dependence on determination of the limiting pressure. According to the proposed method, examples are given of thick plates ' calculation.

Keywords: high-pressure housing, end element, thick plate, supporting wall, radial stresses, tangential stresses, vertical stresses, internal pressure, uniformly distributed load, key, contact layer.

Рассматривается расчет осесимметричных толстых бетонных плит, опертых по конической поверхности. При этом контактная поверхность плиты и конической поверхности выполнена по скользящей схеме, которая допускает возможное смещение плиты по вертикали.

Как правило, толстые плиты служат для восприятия достаточно высоких воздействий в виде равномерно распределенной нагрузки и могут быть использованы в торцевых участках корпусов высокого давления (КВД) ядерных реакторов [1].

Ниже приводится аналитическое решение задачи по определению предельного давления на толстую плиту. Толстая плита имеет шарнирноподвижное закрепление по вертикали и горизон- тали. При принятых условиях опирания плита будет работать по схеме «пробки».

На рис. 1 представлен общий вид толстой плиты, нагруженной равномерно распределенной нагрузкой $P_{0}$. На рис. 2 представлена расчетная схема толстой плиты в плоском деформированном состоянии. Очевидно, что при действии равномерно распределенной нагрузки на толстую плиту до определенной величины у опор формируется толстостенный свод, причем в угловых зонах (см. поз. 2 на рис. 2) верхней части будет наблюдаться всестороннее сжатие $\left(\sigma_{r}=\sigma_{\theta}=\sigma_{z}=-P_{0} ; \tau_{r z}=0\right)[2-4]$.

Для аналитических расчетов толстых плит в пластической стадии их работы принимается расчетная схема торцевого элемента, представленная на рис. 3. 
a)

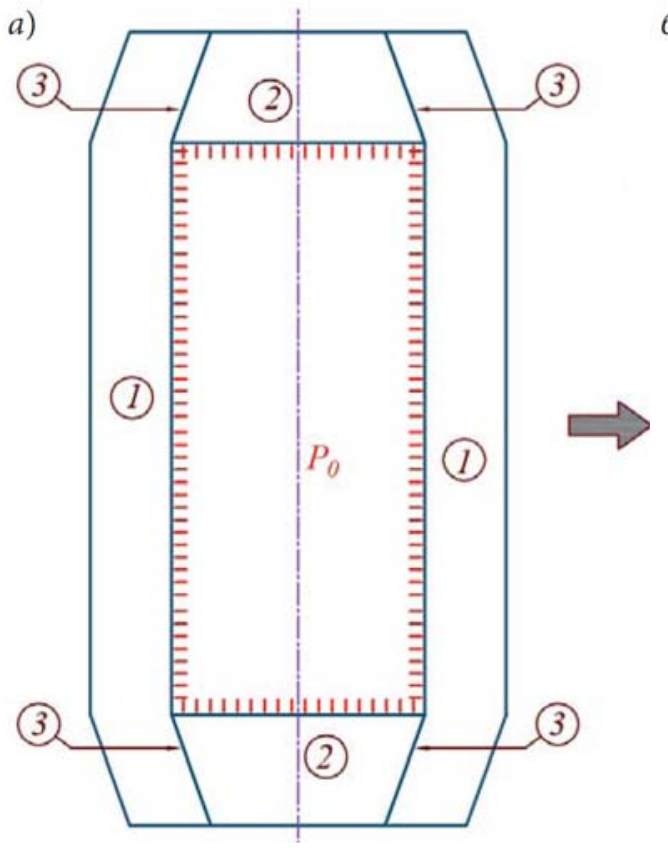

6)

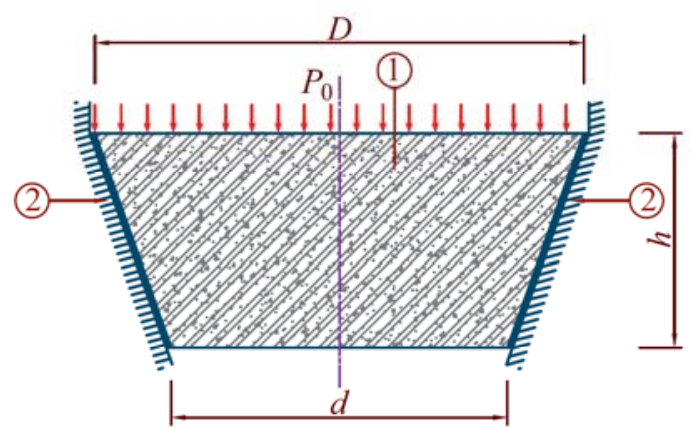

Рис. 1. Общий вид толстой плиты, нагруженной равномерно распределенной нагрузкой $P_{0}$ : $a$ - расчетная схема КВД; 6 - расчетная схема торцевых элементов с трением; 1 - стенка из тяжелого армоцемента; 2 - толстая плита; 3 - контактная поверхность; $P_{0}-$ внутреннее давление

Высота (толщина) свода определяется аналогично методам [2-4], причем точка пересечения $E$ (рис. 4) считается центральной точкой сферического свода, при котором фактически определяются неизвестные параметры свода $a$ и $b$.

Для расчета напряженного состояния толстых плит на основе теории пластичности рассматривается осесимметричная толстая плита, которая представлена в виде сферического свода.

Расчетные формулы для напряженного состояния толстых плит выводятся с применением двух критериев прочности (Баландина и Рихарда - Бранцаега - Брауна), причем принимается, что $\sigma_{\theta}=\sigma_{z}$ как для сферы, при этом купол будем считать полусферой.

Напряженное состояние ториевых элементов с использованием критерия Баландина определим следуюшим образом.

Критерий прочности Баландина [2, 5-7] запишем в виде:

$$
\begin{gathered}
\sigma_{1}^{2}+\sigma_{2}^{2}+\sigma_{3}^{2}-\left(\sigma_{1} \sigma_{2}+\sigma_{2} \sigma_{3}+\sigma_{3} \sigma_{1}\right)- \\
-\left(R_{b}-R_{b t}\right)\left(\sigma_{1}+\sigma_{2}+\sigma_{3}\right)=R_{b} R_{b t} .
\end{gathered}
$$

С учетом $\sigma_{1}=\sigma_{2}=\sigma_{\theta} ; \sigma_{3}=\sigma_{r}$ в соответствии c (1) получим

$$
\left(\sigma_{1}-\sigma_{3}\right)^{2}-\left(R_{b}-R_{b t}\right)\left(2 \sigma_{1}+\sigma_{3}\right)=R_{b} R_{b t} .
$$

Пусть

$$
\begin{gathered}
t=\sigma_{1}-\sigma_{3} \geq 0 ; p=\sigma_{1}+\sigma_{3} ; M=R_{b}-R_{b t} ; \\
N=R_{b} R_{b t} .
\end{gathered}
$$

Совместное решение первых двух выражений дает

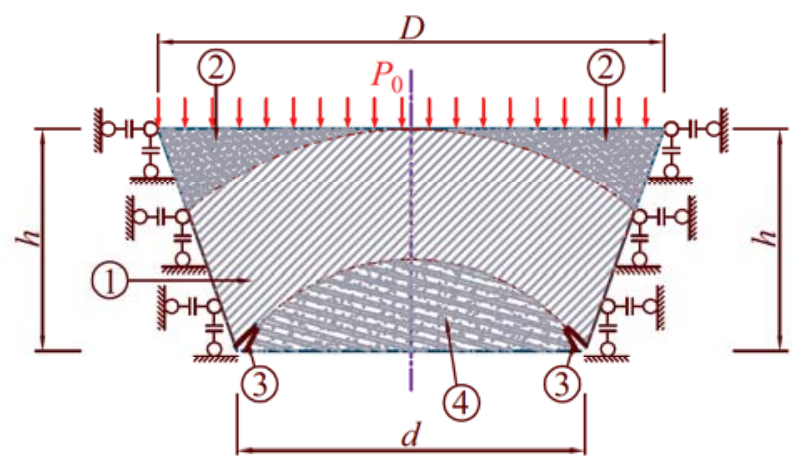

Рис. 2. Расчетная схема толстой плиты при плоском деформированном состоянии: 1 - толстая плита; 2 - зона, где происходит всестороннее сжатие; 3- не рассматривается в данном расчете 


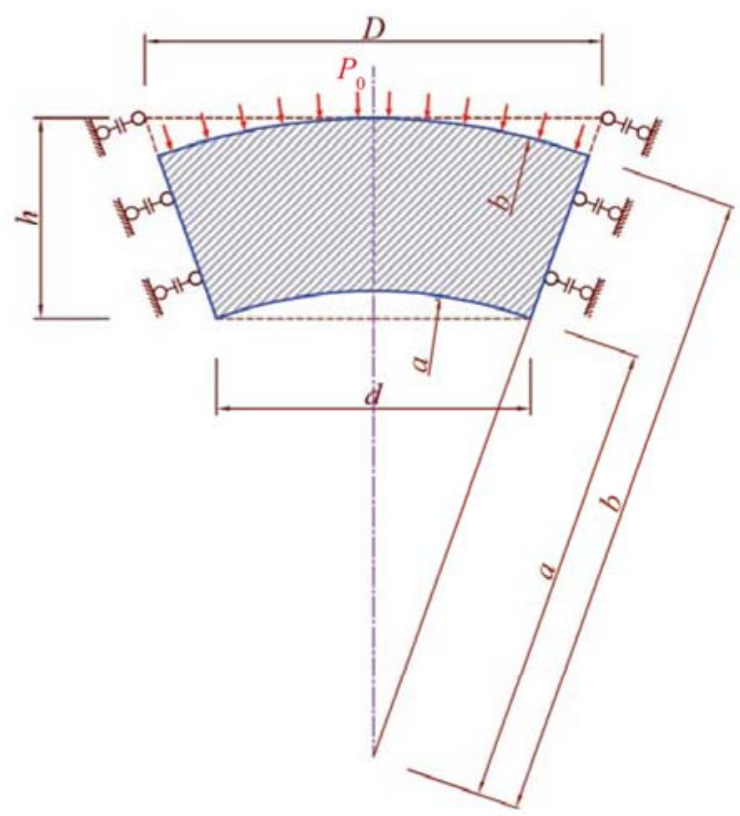

Рис. 3. Расчетная схема торцевого элемента

$$
\sigma_{1}=\frac{p+t}{2} ; \sigma_{3}=\frac{p-t}{2}
$$

С учетом (3) и (4) выражение (2) примет следующий вид:

$$
t^{2}-M\left(p+t+\frac{p-t}{2}\right)-N=0
$$

Из (5) выразим

$$
p=\frac{1}{3 M}\left(2 t^{2}-M t-2 N\right) .
$$

С учетом (6) выражения для напряжений в (4) запишутся как

$$
\begin{gathered}
\sigma_{1}=\frac{1}{3 M}\left(t^{2}+M t-N\right) ; \\
\sigma_{3}=\frac{1}{3 M}\left(t^{2}-2 M t-N\right) .
\end{gathered}
$$

Дифференциальные уравнения равновесия в сферической системе координат применительно к рассматриваемой задаче имеют вид [5-7]:

$$
\frac{d \sigma_{r}}{d r}+2 \frac{\sigma_{r}-\sigma_{\theta}}{r}=0 .
$$

Учитывая, что $\sigma_{3}=\sigma_{r}$, продифференцируем (9):

$$
\frac{d \sigma_{r}}{d r}=\frac{1}{3 M} \frac{(2 t-2 M) d t}{d r} .
$$

С учетом (3) получим:

$$
2 \frac{\sigma_{r}-\sigma_{\theta}}{r}=-\frac{2 t}{r}
$$

С учетом (10) и (11) уравнение равновесия примет вид:

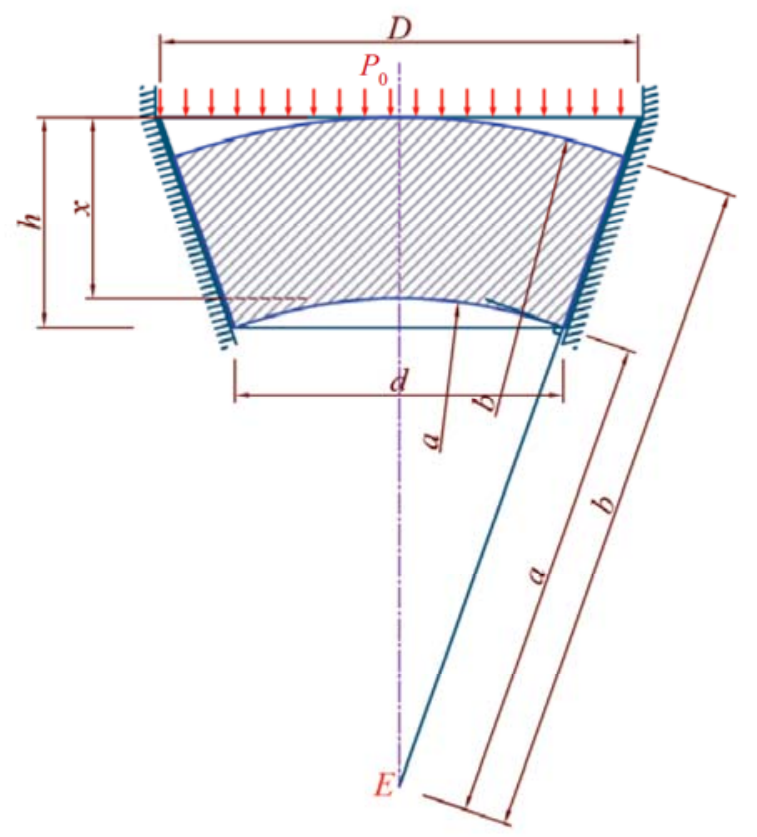

Рис. 4. Определение высоты (толщины) свода: $x$ - толщина свода; $a$ - внутренний радиус; $b-$ внешний радиус

$$
\frac{1}{3 M}\left(1-\frac{M}{t}\right) d t=\frac{d r}{r} .
$$

После интегрирования (12) получим

$$
\frac{t}{M}-\ln t=3 \ln r+c .
$$

Имеем следующие граничные условия для сферы:

$$
\text { При } r=a\left|\begin{array}{c}
t=t_{a} \\
\sigma_{r}=\sigma_{3}=0
\end{array}\right| \text { и } r=b\left|\begin{array}{c}
t=t_{b} \\
\sigma_{r}=\sigma_{3}=P_{0}
\end{array}\right| .
$$

С учетом принятых граничных условий уравнение (13) представим в виде

$$
\left\{\begin{array}{l}
\frac{t_{a}}{M}-\ln t_{a}=3 \ln a+c ; \\
\frac{t_{b}}{M}-\ln t_{b}=3 \ln b+c .
\end{array}\right.
$$

Произведем вычитание двух уравнений (14) с дальнейшим преобразованием:

$$
\begin{gathered}
\frac{t_{b}-t_{a}}{M}-\ln \frac{t_{b}}{t_{a}}=3 \ln \frac{b}{a} \Leftrightarrow \\
\Leftrightarrow \frac{t_{b}-t_{a}}{M}-\ln \left(\frac{t_{b}}{t_{a}}\right)=\ln \left(\frac{b}{a}\right)^{3} \Leftrightarrow \\
\Leftrightarrow \ln \left[\left(\frac{b}{a}\right)^{3}\left(\frac{t_{b}}{t_{a}}\right)\right]=\frac{t_{b}-t_{a}}{M} \Leftrightarrow \\
\Leftrightarrow\left(\frac{b}{a}\right)^{3}=\left(\frac{t_{a}}{t_{b}}\right) e^{\frac{t_{b}-t_{a}}{M}} .
\end{gathered}
$$


Окончательно получим экспоненциальное уравнение напряженного состояния сферь применительно к своду в виде

$$
\left(\frac{b}{a}\right)^{3}=\left(\frac{t_{a}}{t_{b}}\right) e^{\frac{t_{b}-t_{a}}{M}} .
$$

При $r=a, \sigma_{r}=\sigma_{3}=0$ из формулы (8) получим уравнение

Откуда

$$
t_{a}^{2}-2 M t_{a}-N=0 .
$$

$$
t_{a}=M+\sqrt{M^{2}+N} .
$$

Полученное значение $t_{a}$ подставим в (15) и найдем $t_{b}$.

При $\sigma_{r}=\sigma_{3}=P_{0}$ и с учетом $t_{b}$ по формуле (8) получим предельную величину давления

$$
P_{0}=\frac{1}{3 M}\left(t_{b}^{2}-2 \cdot M \cdot t_{b}-N\right) .
$$

Отметим, что оригинальное уравнение напряжений для сферы (15) решается с учетом параметра $t_{a}$ и с использованием численного метода MATHCAD 15, откуда определяется параметр $t_{b}$.

В качестве иллюстрации использования полученных зависимостей ниже приводится пример расчета.

Исходные данные толстой плиты для бетона B40 [2]: $R_{b}=22 \mathrm{M \Pi а}, R_{b t}=1,4 \mathrm{M \Pi а}, d=0,587 \mathrm{~m}$, $h=0,75 \mathrm{M}$.

В зависимости от $x$ графически определяем параметры:

$a=1746$ мм; $b=2385$ мм; $t_{a}=41,9 ; t_{b}=72,5$.

Для практического расчета напряженного состояния толстой плиты $\left(\sigma_{r} ; \sigma_{\theta}\right)$ используется совместное решение уравнений (15), (8) и (7).

По формуле (18) определяем величину максимального давления: $P_{0}=35 \mathrm{MПа.}$

В табл. 1 и на рис. 5 и 6 приведены некоторые результаты решения задачи расчета НДС и прочности, выполненного на основе критерия Баландина.

Далее определим напряженное состояние ториевых элементов на основе критерия Ф. Рихарда, А. Бранцаега и Р. Брауна.
Для определения главных напряжений $\left(\sigma_{\theta}=\sigma_{\varphi}=\sigma_{1}=\sigma_{2}\right.$ и $\left.\sigma_{r}=\sigma_{3}\right)$ используется дифференциальное уравнение равновесия в сферической системе координат [5-7]:

$$
\frac{d \sigma_{3}}{d r}+2 \frac{\sigma_{3}-\sigma_{1}}{r}=0,
$$

где $\sigma_{1}=\sigma_{\theta}-$ максимальное значение напряжений; $\sigma_{3}=\sigma_{r}-$ минимальное значение напряжений.

На основе экспериментальных данных [8] за величину максимальных напряжений $\sigma_{1}$ принимается уровень напряжений, при которых начинают образоваться магистральные трещины (рис. 8) из [8]:

$$
\sigma_{1}=R_{b}^{\prime \prime \prime} .
$$

Для определения прочности бетона при трехосном сжатии $R_{b}^{\prime \prime \prime}$ используется критерий прочности Рихарда - Бранцаега - Брауна [9]:

$$
R_{b}^{\prime \prime \prime}=R_{b}+k \cdot \sigma_{\mathrm{o}}
$$

где $k-$ коэффициент эффективности бокового давления; $R_{b}-$ прочность бетона при одноосном сжатии; $\sigma_{\mathrm{o}}$ - гидростатическое обжатие бетона.

Из (20) и (21) следует

$$
\sigma_{1}=R_{b}+k \cdot \sigma_{3} .
$$

Граничные условия имеют вид:

$$
\begin{gathered}
\left.\sigma_{r}\right|_{r=a}=0 \\
\left.\sigma_{r}\right|_{r=b}=P_{0}
\end{gathered} .
$$

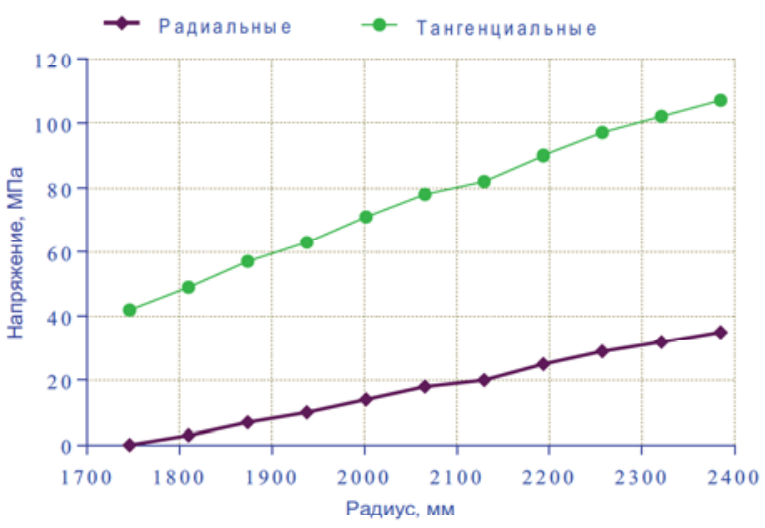

Рис. 5. График зависимости тангенциального и радиального напряжения от радиуса свода

Таблица 1

Радиальные и тангенциальные напряжения в зависимости от радиуса $r$

\begin{tabular}{|c|c|c|c|c|c|c|c|c|c|c|c|}
\hline \multicolumn{1}{|c}{$r$, мм } & 1746 & 1810 & 1874 & 1938 & 2002 & 2066 & 2129 & 2193 & 2257 & 2321 & 2385 \\
\hline$\sigma_{r}$, МПа & 0 & 3 & 7 & 10 & 14 & 18 & 20 & 25 & 29 & 32 & 35 \\
\hline$\sigma_{\theta}$, МПа & 42 & 49 & 57 & 63 & 71 & 78 & 82 & 90 & 97 & 102 & 107 \\
\hline
\end{tabular}



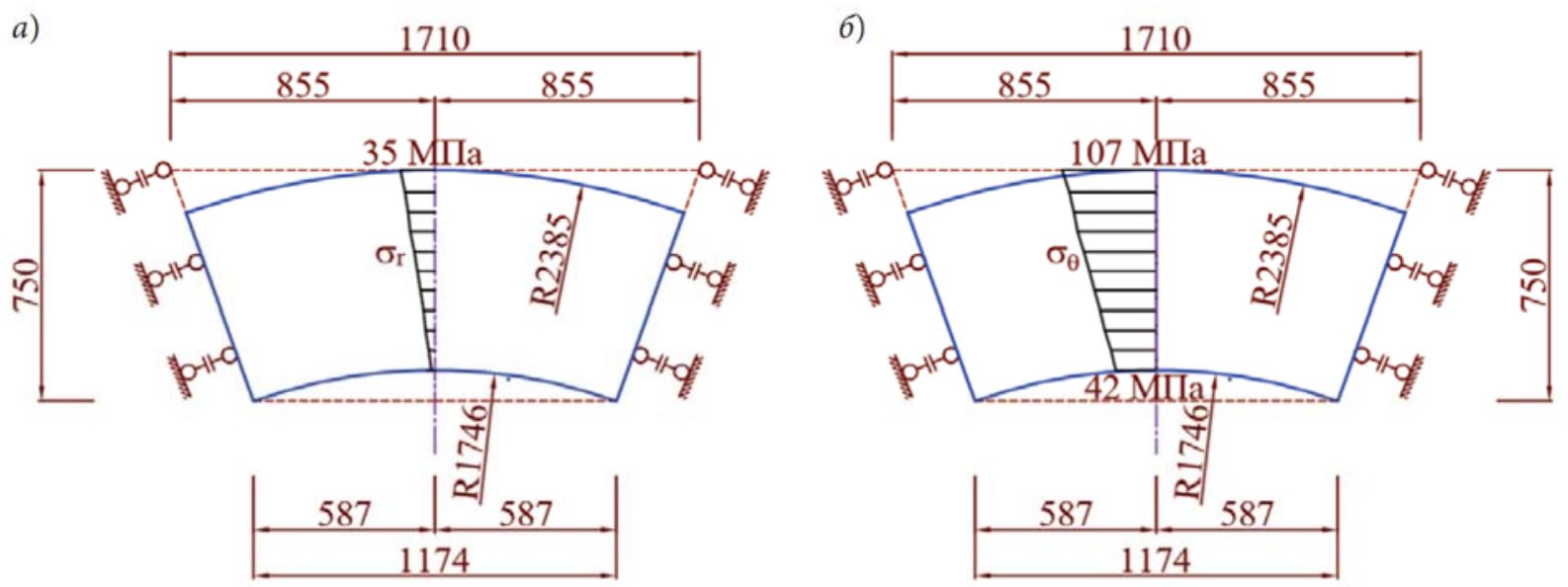

Рис. 6. Результаты расчетов напряженного состояния: $a$ - радиальные; 6 - тангенциальные напряжения

Пусть $p=\frac{\sigma_{1}+\sigma_{3}}{2} ; t=\frac{\sigma_{1}-\sigma_{3}}{2}$.

Тогда

$$
\sigma_{1}=p+t ; \sigma_{3}=p-t .
$$

Подставим (23) в критерий (21):

$$
(1-k) p+(1+k) t-R_{b}=0 .
$$

Теперь напряжения можно записать в виде

$$
\sigma_{1}=\frac{R_{b}}{1-k}-\frac{2 \cdot k}{1-k} t ; \sigma_{3}=\frac{R_{b}}{1-k}-\frac{2}{1-k} t
$$

Подстановка (24) в условия статики (19) дает

$$
\frac{d t}{d r}-2(k-1) \frac{t}{r}=0 .
$$

Решение (25) с учетом граничных условий позволяет выразить через (24)

$$
\begin{gathered}
t_{b}=\left(\frac{R_{b}}{1-k}-P_{0}\right) \frac{1-k}{2} ; t_{a}=\frac{R_{b}}{2} ; \\
P_{0}=\frac{R_{b}}{3}\left[\left(\frac{b}{a}\right)^{6}-1\right] .
\end{gathered}
$$

Принято $k=4$.

Для практического использования полученной формулы ниже приводится пример расчета.

Исходные данные толстой плиты [2] для бетона В40: $R_{b}=22 \mathrm{MПа,} R_{b t}=1,4 \mathrm{MПа,} d=587 \mathrm{мм}$, $h=750 \mathrm{MM}$.
Определяем параметры: $a=1746$ мм; $b=2385$ мм.

Для практического расчета напряженного состояния $\left(\sigma_{r} ; \sigma_{\theta}\right)$ используются формулы (24), (26).

Максимальное давление $P_{0}=40$ МПа.

В табл. 2 и на рис. 7 и 8 приведены некоторые результаты решения задачи о НДС и прочности, выполненного на основе критерия Ф. Рихарда, А. Бранцаега и Р. Брауна.

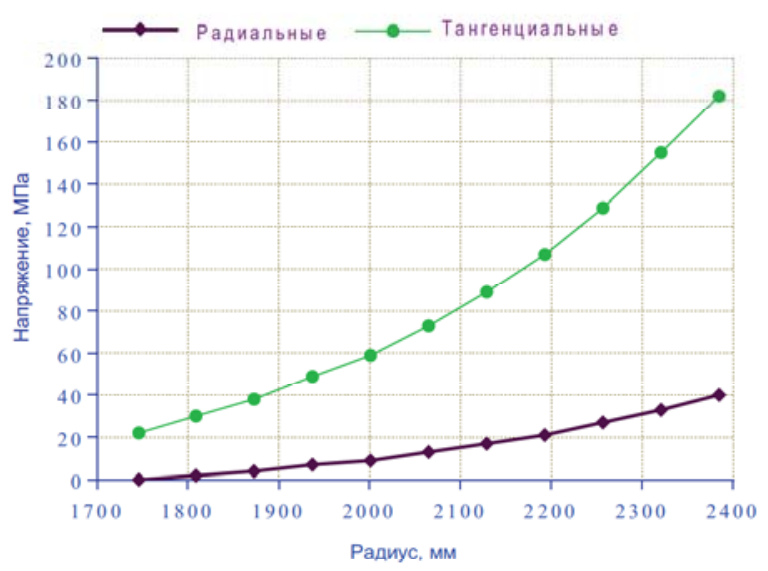

Рис. 7. График зависимости тангенциального и радиального напряжений от радиуса свода

Таблица 2

Радиальные и тангенциальные напряжения в зависимости от радиуса $r$

\begin{tabular}{|c|c|c|c|c|c|c|c|c|c|c|c|}
\hline$r$, мм & 1746 & 1810 & 1874 & 1938 & 2002 & 2066 & 2129 & 2193 & 2257 & 2321 & 2385 \\
\hline$\sigma_{r}$, МПа & 0 & 2 & 4 & 7 & 9 & 13 & 17 & 21 & 27 & 33 & 40 \\
\hline$\sigma_{\theta}$, МПа & 22 & 30 & 38 & 49 & 59 & 73 & 89 & 107 & 129 & 155 & 182 \\
\hline
\end{tabular}



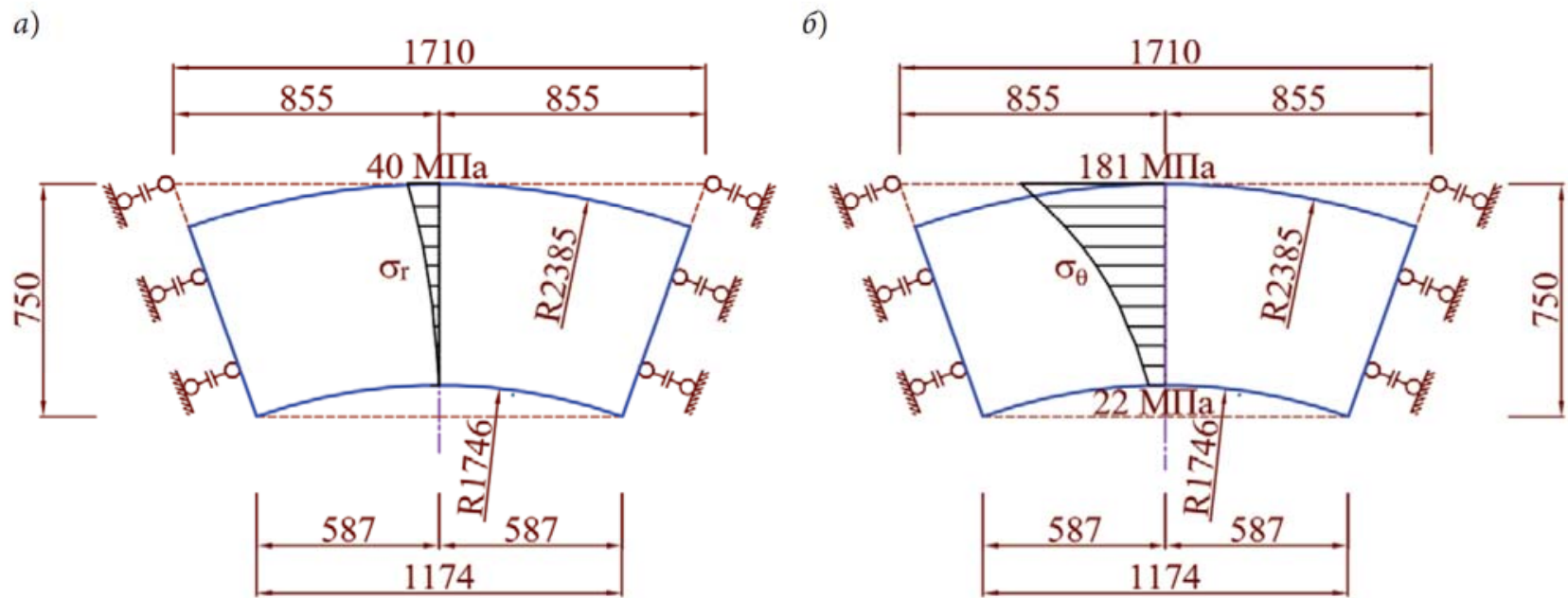

Рис. 8. Результаты расчетов напряженного состояния: $a$ - радиальные; 6 - тангенциальные напряжения

Таким образом, выполнен аналитический расчет напряженного состояния и предельных нагрузок толстых плит, свободно опертых по конической поверхности. Полученные результаты показывают адекватность предлагаемой методики расчета.

\section{Библиографический список}

1. Morozov V., Pucharenko Ju. Nuclear Reactor Shell of Heavy Ferrocement // World Applied Sciences Journal (Problems of Architecture and Construction). 2013. Vol. 23. Pp. 31-36.

2. Морозов В. И. Корпуса высокого давления для энергетических, строительных и специальных технологий. СПб.: СПбГАСУ, 2011. 394 с.

3. Морозов В. И., Повышев Н. И., Буриев В. М. Толстостенные железобетонные сосуды при силовых и тепловых воздействиях // Строительные конструкции зданий и сооружений: межвуз. сб. Барнаул: АПИ, 1989. С. 61-71.

4. Писаренко Г. С., Амельянович К. К., Каринцев И. Б. Несущие и светопрозрачные элементы конструкций из стекла. Киев: Наукова думка, 1987. 198 с.

5. Самуль В. И. Основы теории упругости и пластичности. М.: Высшая школа, 1982. 263 с.

6. Гениев Г. А., Киссюк В. Н., Тюпин Г. А. Теория пластичности бетона и железобетона. М.: Стройиздат, $1974.306 \mathrm{c}$.

7. Тимошенко С. П., Войновский-Кригер С. Пластинки и оболочки. М.: Наука, 1966. 636 с.

8. Зайцев Ю. В., Леонович С. Н., Шнайдер У. Структура, прочность и механика разрушения бетонов при двухосном и трехосном сжатии. Минск: БНТУ, 2011. $382 \mathrm{c}$.
9. Richart F. E., Brandtzaeg A., Brown R. L. A study of the failure of concrete under combined compressive stresses. (Technical Report Bulletin No. 185) Engineering Experiment Station, University of Illinois, Urbana; 1928.

\section{References}

1. Morozov V., Pucharenko Ju. Nuclear Reactor Shell of Heavy Ferrocement. World Applied Sciences Journal (Problems of Architecture and Construction), 2013, vol. 23, pp. 31-36.

2. Morozov V. I. Korpusa vysokogo davleniya dlya ehnergeticheskikh, stroitel'nykh i spetsial'nykh tekhnologiy [High-pressure shells for power, construction and special technologies]. St. Petersburg, SPSUACE Publ., 2011, 394 p.

3. Morozov V. I., Povyshev N. I., Burtsev V. M. Tolstostennye zhelezobetonnye sosudy pri silovykh $i$ teplovykh vozdeystviyakh [Thick-walled reinforced concrete vessels at power and thermal influences]. Mezhvuz. sb «Stroitel'nye konstruktsii zdaniy i sooruzheniy» [Interhigher education institution coll. of works "Building constructions of buildings and structures"]. Barnaul, API Publ., 1989, pp. 61-71.

4. Pisarenko G. S., Amel'yanovich K. K., Karintsev I. B. Nesushchie i svetoprozrachnye ehlementy konstruktsiy iz stekla [Bearing and translucent elements of glass designs]. Kiev, Naukova dumka Publ., 1987, 198 p.

5. Samul' V. I. Osnovy teorii uprugosti i plastichnosti [Basics of the elasticity and plasticity theory]. Moscow, Vysshaya shkola Publ., 1982, 263 p.

6. Geniev G. A., Kissyuk V. N., Tyupin G. A. Teoriya plastichnosti betona $i$ zhelezobetona [The theory of plasticity of concrete and reinforced concrete]. Moscow, Stroyizdat Publ., 1974, 306 p. 
7. Timoshenko S. P., Voynovskiy-Kriger S. Plastinki i obolochki [Plates and covers]. Moscow, Nauka Publ., 1966, $636 \mathrm{p}$.

8. Zaytsev Yu. V., Leonovich S. N., Shnayder U. Struktura, prochnost' i mekhanika razrusheniya betonov pri dvukhosnom i trekhosnom szhatii [Structure, durability and fracture mechanics of concrete at two-axis and threeaxis compression]. Minsk, BNTU Publ., 2011, 382 p.

9. Richart F. E., Brandtzaeg A., Brown R. L. A study of the failure of concrete under combined compressive stresses. (Technical Report Bulletin no. 185). Engineering Experiment Station Publ., University of Illinois, Urbana; 1928. 